\title{
Originalien
}

Rechtsmedizin 2020 · 30:71-78

https://doi.org/10.1007/s00194-020-00371-7

Online publiziert: 25. Januar 2020

(c) Der/die Autor(en) 2020

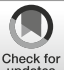

S. Heinze ${ }^{1} \cdot$ S. Grunert ${ }^{2} \cdot$ S. Grabherr ${ }^{3} \cdot$ D. Labudde ${ }^{2} \cdot$ Arbeitsgemeinschaft $^{2}$ Forensische Bildgebung (AGFB) der Deutschen Gesellschaft für Rechtsmedizin (DGRM)

${ }^{1}$ Institut für Rechts- und Verkehrsmedizin, Universitätsklinikum Heidelberg, Heidelberg, Deutschland

${ }^{2}$ FoSIL (Forensic Science Investigation Lab), Hochschule Mittweida, Mittweida, Deutschland

${ }^{3}$ CURML - Universitätszentrum für Rechtsmedizin Lausanne-Genf, Lausanne-Genf, Schweiz

\section{Erstellung und Etablierung einer wissensbasierten Fallsammlung für forensische Bildgebung - WiFas}

\section{Einleitung}

Mit der Weiterentwicklung und Verbreitung radiologischer Techniken wie Computertomographie (CT) und Magnetresonanztomographie (MRT) hat in den letzten 2 Jahrzehnten die forensische Bildgebung einen immer größeren Stellenwert sowohl in der postmortalen als auch in der klinischen Rechtsmedizin eingenommen $[1-4,7-9,11,12$, 14-16]. Um diese neuen Techniken und die damit verbundenen Möglichkeiten in der Rechtsmedizin gezielt einzusetzen und in Deutschland zu verbreiten, wurde im Rahmen der 93. Jahrestagung der Deutschen Gesellschaft für Rechtsmedizin (DGRM) in Heringsdorf 2014 die Arbeitsgemeinschaft für forensische Bildgebung (AGFB) gegründet. Eines der Ziele dieser Arbeitsgemeinschaft ist die Ausbildung der Personen, die für die Interpretation der rechtsmedizinisch generierten radiologischen Daten zuständig sind. Um solche Daten allen Mitgliedern der DGRM zugänglich zu machen, und zwar unabhängig davon, ob im eigenen Institut bereits eine Bildgebung angewendet wird oder nicht, begann die AGFB im Jahr 2015 mit der Entwicklung der Wissensbasierten Fallsammlung (WiFas) [5].

Mit der WiFas sollte erstmals eine Plattform angeboten werden, auf deren Grundlage verschiedenste Informationen aus den Bereichen der Rechtsmedi- zin und der Radiologie zusammenfließen können. Ziel des Projekts war es, den Mitgliedern sowohl die Möglichkeit zu geben, anhand einzelner Fälle zu lernen und zu lehren, als auch gezielt Fälle miteinander $\mathrm{zu}$ vergleichen.

\section{Material und Methoden}

\section{Konzeption und Implementierung des Systems}

Das vorliegende System wurde auf Basis von PHP, einer Skriptsprache mit einer an C und Perl angelehnten Syntax (ursprünglich: Personal Home Page Tools), die hauptsächlich zur Erstellung dynamischer Webseiten oder Webanwendungen verwendet wird, entwickelt, um die Erstellung und Verwaltung rechtsmedizinischer Fälle zu ermöglichen [13].

Im Vordergrund stand die Abbildung einer fachgebietsspezifischen Prozesskette, in der für die Teilbereiche wie z. B. Bildgebung, klinische Untersuchung, Leichenschau, Sektion sowie Fundort Sachverhalte innerhalb von Einzelgutachten abgebildet werden können. Um in diesem Prozess externe Dokumente, Bilder und Dateiarchive zu Einzelgutachten hinzuzufügen und zu verwalten, wurden fest definierte Schnittstellen zur Verfügung gestellt. Alle in diesem Zusammenhang stehenden Daten wurden durch ein freies, objektrelationales Datenbankmanagementsystem, eine PostgreSQL-Datenbank (https:// www.postgresql.org/), verwaltet. Für die persistente Datenspeicherung wurde bis jetzt auf die Version 12 von PostgreSQL zurückgegriffen.

Aktuell werden das WiFas-System und alle in diesem Zusammenhang stehenden Daten durch das Forensic Sciences Investigation Lab (FoSIL) der Hochschule Mittweida auf einer eigens dafür in Betrieb genommenen ServerHardware-Infrastruktur gehostet und betrieben. Sowohl die hardware- als auch softwareseitige Administration unterliegt dabei dem FoSIL.

\section{Gestaltung der Suchmasken}

Insgesamt 3 Eingabemasken wurden zur Verfügung gestellt, um die gezielte Suche nach bereits erstellten Fällen zu unterstützen.

Es wurde sowohl eine einfache $\mathrm{Su}$ che, die Fälle, basierend auf bestimmten Schlagwörtern, als auch eine grafische und detaillierte Suche in der PostgreSQLDatenbank implementiert. Dabei wurde das Auswählen interaktiver Körperregionen auf einem anatomischen Modell bzw. durch UND-Verknüpfungen von Schlagworten zu den Themen Anatomie, Ursache und Fundort für den Datenbanknutzer ermöglicht.

Suchanfragen wurden so programmiert, dass diese ausschließlich über PostgreSQL (eine Datenbanksprache, 

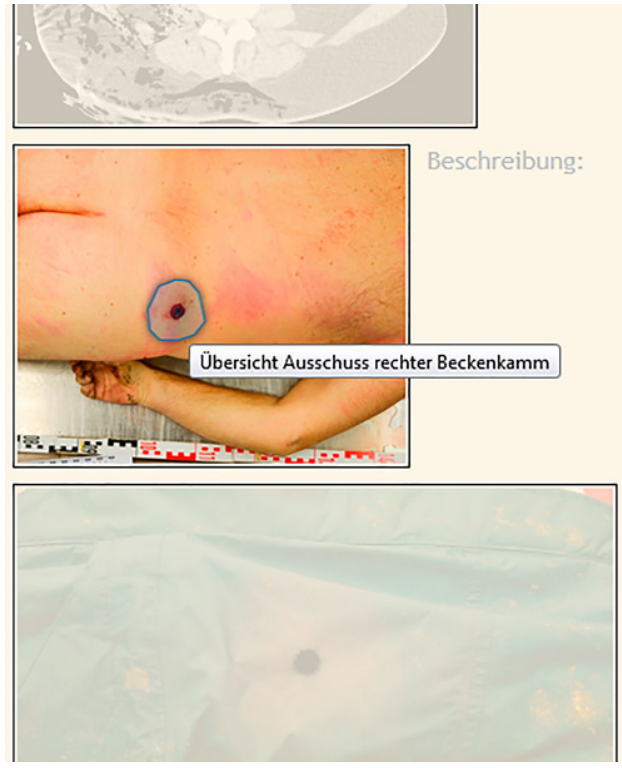

Beschreibung:

Abb. $1<$ Annotationen in einer hochgeladenen Fotografie

auf deren Grundlage die Kommunikation wie z. B. das Bearbeiten und Abfragen von Datenbeständen) mit der zugrunde liegenden PostgreSQL-Datenbank erfolgen. Eine sukzessive Optimierung des Datenbanksystems umfasste den Einsatz von Indizes, die Analyse und Optimierung von Abfragen, die Reorganisation sowie die optimale Anpassung und Nutzung zur Verfügung stehender Systemressourcen.

\section{Aufbau der Falleinstellung in die Datenbank}

Für den „content upload“ wurden innerhalb des „user interface“ (UI, englisch für Benutzerschnittstelle) zahlreiche Möglichkeiten zur Verfügung gestellt, um die Systemintegration diverser Dateien, unterschiedlicher Typen, zu gewährleisten. Bisher wurde dabei auf Version 5.2.0 von DropzoneJS (https://www.dropzonejs. $\mathrm{com} /$ ), eine Open-Source-Bibliothek für das Hochladen von Dateien per Drag \& Drop mit integrierter Bildvorschau, zurückgegriffen.

Auf Grundlage der in der Programmiersprache JavaScript entwickelten Open-Source-Bibliotheken CornerstoneJS (https://cornerstonejs.org/), von Chris Hafey, und jQuery (https://jquery. $\mathrm{com} /$ ) wurde es ermöglicht, nach erfolgtem Upload Bilddateien mit Annotationen $\mathrm{zu}$ versehen und somit näher $\mathrm{zu}$ beschreiben (unter einer Annotation ist an dieser Stelle ein markierter Bereich auf einem Bild $\mathrm{zu}$ verstehen, der zusätzlich textuell näher beschrieben werden kann (- Abb. 1)). Das Durchsuchen, Bearbeiten sowie das Animieren von HTMLDokumenten wurde mittels jQuery, das über eine benutzerfreundliche Programmierschnittstelle, eine „application programming interface (API)“, verfügt und für eine Vielzahl von Browsern unterstützt wird, ermöglicht.

\section{Implementierung eines Viewers für Bilddaten}

Zur Unterstützung eines robusten Parsens von DICOM(Digital Imaging and Communications in Medicine)-Dateien, den in diesem Zusammenhang stehenden Übertragungssyntaxen sowie einer „Multi-threaded“-Bilddecodierung wurde CornerstoneJS gewählt.

Der modulare Aufbau von CornerstoneJS ermöglichte die einfache Entwicklung eigener Werkzeuge zur Interaktion mit DICOM-Dateien. Auch eine anschauliche Visualisierung medizinischer Daten im DICOM-Format sowie anderer Bildformate ${ }^{*}$.gif, ${ }^{*}$.jpg, ${ }^{*}$.png etc.), wurde auf Grundlage von CornerstoneJS und einem systeminternen Bildbetrachter in die Datenbank integrierbar. Dies macht es möglich, dass innerhalb dieses Bildbetrachters komplette DICOM-Bildarchive näher analysiert und mithilfe unterschiedli- cher Funktionen aus der CornerstoneJSBibliothek skaliert, gespiegelt, rotiert, interpoliert, invertiert, vermessen oder exportiert werden können.

\section{Systemzugriff und Rollen- verteilung}

Jeder WiFas-Nutzer verfügt über ein systeminternes Nutzerkonto. Damit wird ein unautorisierter Systemzugriff von außen, durch unbefugte Personen, verhindert. Ein Nutzer-Rollen-Konzept trägt dazu bei, den systeminternen Informationszugriff zu steuern. Dabei ist ein Nutzerkonto, der personalisierte Systemzugang, mit einer bestimmten Rolle gekoppelt. Das bedeutet, dass ein Nutzer innerhalb des WiFas-Systems eine gewisse Rolle einnimmt, in der er über unterschiedliche Rechte verfügt. Diese Rechte gewährleisten ein Management bei Erstellen, Bearbeiten, Löschen und Exportieren fallspezifischer Daten und schließlich des gesamten systeminternen Informationszugriffs. Die Rollen- bzw. Rechtevergabe ist systemintern fest vorgeschrieben. Eine Änderung der Rechte einer Rolle würde demzufolge alle Nutzer betreffen, welche die zuvor geänderte Rolle besitzen. Neue Rollen können hinzugefügt werden und bereits existierende ggf. geändert oder gelöscht werden. Die Administration einzelner Rollen und deren Vergabe unterliegen dabei den Autoren. Nach erfolgter Registrierung und dem Einloggen in das WiFas-System (s. Abschn. „Arbeiten mit der WiFas, Einloggen“) verfügt ein Nutzer grundlegend über alle Rechte, welche das Management eigener fallspezifischer Daten gewährleisten. Erweiterte Rollen z.B. zur Prüfung fallspezifischer Daten im Rahmen eines Review-Prozesses (s. Abschn. „Ergebnisse - Etablierung der WiFas"), sowie Rollen zur systeminternen administrativen Verwaltung, unterliegen den Autoren. Die Rolle zur Prüfung fallspezifischer Daten im Rahmen eines Review-Prozesses kann fallspezifisch übertragen werden.

\section{Anonymisierung}

Von Beginn des Etablierungsprozesses der WiFas an stand die Anonymisierung der systemintern verwalteten Daten 
Rechtsmedizin 2020 · 30:71-78 https://doi.org/10.1007/s00194-020-00371-7

(c) Der/die Autor(en) 2020

S. Heinze · S. Grunert · S. Grabherr · D. Labudde · Arbeitsgemeinschaft Forensische Bildgebung (AGFB) der Deutschen Gesellschaft für Rechtsmedizin (DGRM)

\section{Erstellung und Etablierung einer wissensbasierten Fallsammlung für forensische Bildgebung - WiFas}

Zusammenfassung

Einleitung. Mit der Weiterentwicklung und Verbreitung radiologischer Techniken wie Computertomographie und Magnetresonanztomographie hat in den letzten 2 Jahrzehnten die forensische Bildgebung einen immer größeren Stellenwert eingenommen. Um die damit verbundenen Ergebnisse und daraus gewonnenen Erkenntnisse sowie die in diesem Zusammenhang stehenden radiologischen Daten, welche diese neuen Techniken hervorbringen, allen Mitgliedern der Deutschen Gesellschaft für Rechtsmedizin zugänglich zu machen, wurde die Wissensbasierte Fallsammlung (WiFas) entwickelt.

Material und Methoden. Die Datenbank wurde als Webapplikation entwickelt. Es können externe Dokumente, Bilder und Dateiarchive zu Einzelgutachten in die
Datenbank geladen und verwaltet werden. Ein systeminternes Nutzer-Rollen-Konzept verhindert den unautorisierten Systemzugriff durch unbefugte Personen.

Ergebnisse. Es ist eine Datenbank entstanden, die jedem Nutzer einen individuellen Zugang zu zahlreichen rechtsmedizinischen Fällen erlaubt. In der WiFas ist es möglich, sowohl eine Übersicht an Fällen zu erhalten, als auch gezielt nach einzelnen Fallarten zu suchen und Befunde in verschiedenen Dokumenten und Bildmaterial zu vergleichen.

Diskussion. Sowohl die klinische forensische Bildgebung als auch die postmortale Bildgebung sind nicht mit der klinischen Radiologie gleichzusetzen. Oftmals werden seitens der Ermittlungsbehörden rekonstruktive Fragen gestellt, deren Beantwortung nicht nur radiologisches Wissen, sondern auch rechtsmedizinisches Fachwissen fordert. Außerdem sind häufig spezifische Darstellungen der Bilddaten (z. B. dreidimensionale Darstellungen von Befunden) erforderlich. Im gesamten Gebiet der forensischen Bildgebung ist dementsprechend interdisziplinäres radiologisches und rechtsmedizinisches Wissen notwendig. Mit der WiFas wird erstmals eine Plattform angeboten, auf deren Grundlage verschiedenste Informationen aus den Bereichen der Rechtsmedizin und der Radiologie zusammenfließen können.

Schlüsselwörter

Forensische Bildgebung - Datenbank . Interaktives Lernen · Postmortale Bildgebung · Forensische Radiologie

\section{Compilation and establishment of a knowledge-based case databank for forensic imaging-WiFas}

\section{Abstract}

Introduction. During the last 20 years forensic imaging became increasingly more important due to the wider distribution and advancements of radiological techniques, such as computed tomography and magnetic resonance imaging. The databank on a knowledge-based case collection (WiFas) was developed to make the results and knowledge gained from these new techniques and the radiological data obtained available for all members of the German Society of Legal Medicine.

Material and methods. The databank was developed as a web application. It is possible to upload and manage external documents, pictures and datasets from individual case reports. An internal user profile concept prevents access to the system by unauthorized persons.

Results. A databank was developed that enables users to have an individual access to numerous forensic cases. In the WiFas it is possible to obtain an overview of the cases as well as to search for single cases. Furthermore, findings from different cases and images can be compared.

Conclusion. Clinical forensic imaging and post-mortem imaging cannot be equated with clinical radiology. Investigative authorities often ask additional reconstructive questions and answering these questions needs not only forensic but also radiological specialized knowledge. Furthermore, specific or new presentations of the imaging datasets (e.g. three-dimensional representation of findings) are often necessary. For the subspecialty of forensic imaging, appropriate interdisciplinary radiological as well as forensic knowledge is necessary. This knowledge-based databank for forensic imaging is the first platform that enables the combination of very different information from the fields of radiology and forensic medicine.

\section{Keywords}

Forensic imaging · Databank - Interactive learning · Post-mortem imaging · Forensic radiology im Vordergrund. Die Anonymisierung wurde daher direkt nach dem Upload jeglicher Dateitypen implementiert, in dem der ursprüngliche Dateiname durch einen zuvor randomisiert erstellten 12stelligen Dateinamen ersetzt wurde. Für die Anonymisierung patientenspezifischer, medizinischer DICOMDaten, welche personenspezifische Informationen wie z.B. Name, Identifizierungsnummer oder Geburtsdatum sowie weitere personenspezifische Zeit- und Datumsangaben, auf der Metaebene enthalten können, wurde an den eigentlichen Datei-Upload-Prozess ein weiterer Anonymisierungsschritt angeschlossen (• Abb. 2). Des Weiteren wurde unabhängig vom Upload-Prozess eine zeitbasierte Ausführung von Anonymisierungsprozessen auf dem zugrunde liegenden Debian-Linux-Betriebssystem mittels sogenannter Cronjobs eingeführt.

\section{Fallsammlung}

Um die erstellte Plattform mit ersten Fällen zu füllen, luden Mitglieder der AGFB eigene, interessante bereits abgeschlossene Fälle in anonymisierter Form in die Datenbank hoch. Hierzu wurde u. a. zusätzlich zu den 2-mal im Jahr stattfindenden Arbeitsgemeinschaftstreffen ein Workshop im FoSIL der Hochschule Mittweida organisiert, sodass gemeinsam an zahlreichen Computern Fälle 


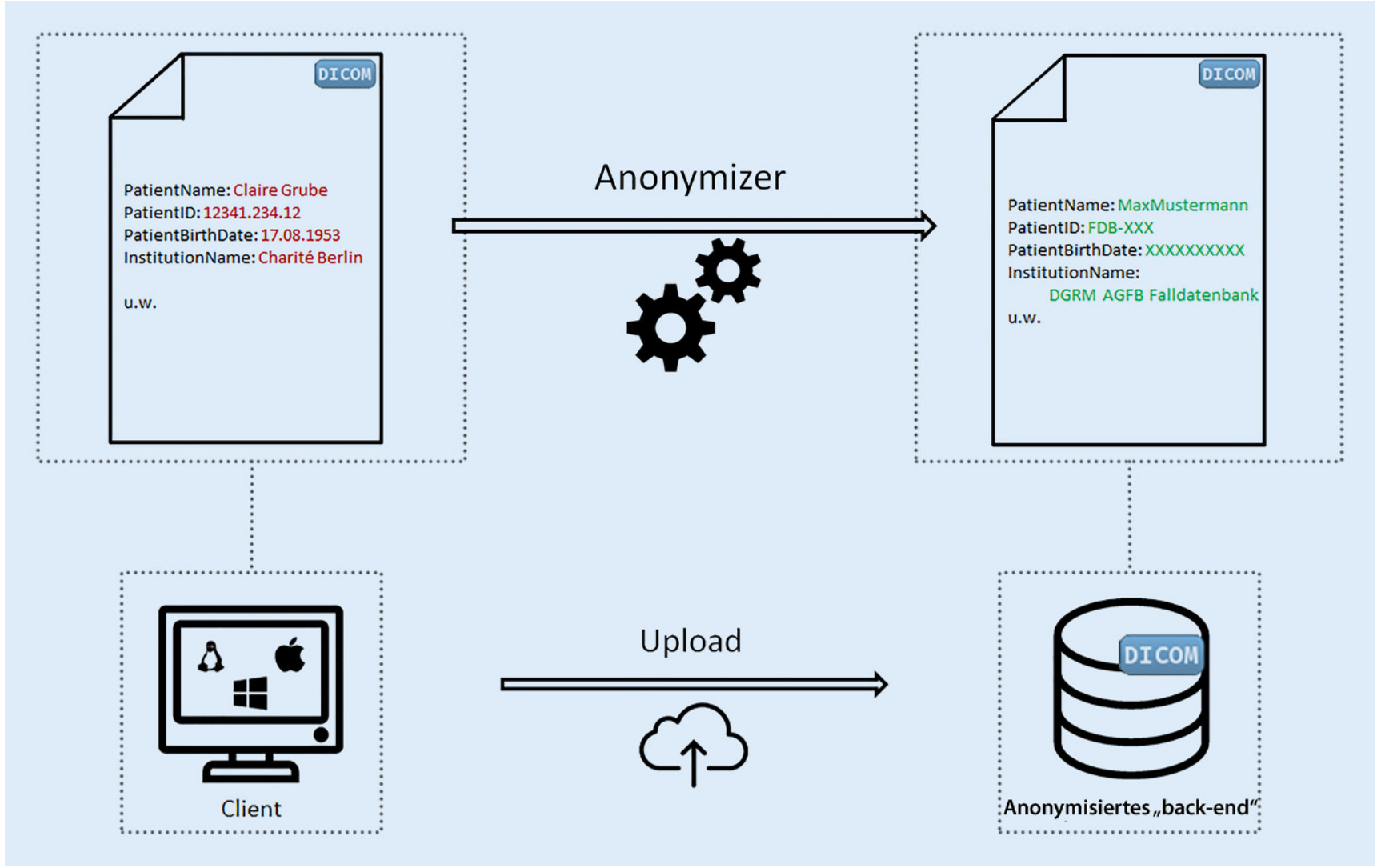

Abb. 2 A Anonymisierung von DICOM(Digital Imaging and Communications in Medicine)-Daten. Nach erfolgtem DateiUpload schließt sich ein Anonymisierungsschritt an, in dem patientenspezifische Informationen anonymisiert werden

von den AGFB-Mitgliedern eingestellt werden konnten. Nach der Komplettierung des Hochladens eines Falls wurde dieser an die Editoren geschickt. Bevor die Freigabe erfolgte, wurde jeder Fall fachärztlich rechtsmedizinisch und radiologisch im Review-System überprüft. Erst nach erfolgter Freigabe wurde dieser Fall für alle Benutzer der Datenbank sichtbar.

\section{Ergebnisse}

\section{Etablierung der WiFas}

Dank der kontinuierlichen Zusammenarbeit der Mitglieder der AGFB und Weiterentwicklung der Prozesse ist aktuell eine Datenbank entstanden, die jedem Nutzer einen individuellen Zugang zu zahlreichen rechtsmedizinischen Fällen mit entweder klinischer oder postmortaler Bildgebung erlaubt.

Zum Zeitpunkt der Artikelerstellung befinden sich in der WiFas 32 Fälle. Von diesen sind 15 vollständig für alle Nutzer freigegeben. Des Weiteren befinden sich 17 von Nutzern hochgeladene Fälle in Bearbeitung bzw. im Review-Prozess.

Der Review-Prozess ist analog zu wissenschaftlichen Fachzeitschriften aufgebaut worden. Mindestens ein/eine rechtsmedizinischer/rechtsmedizinische Facharzt/Fachärztin und ein/eine radiologischer/radiologische Facharzt/ Fachärztin, die jeweils entsprechende Erfahrung in der forensischen Bildgebung besitzen, begutachten jeden Fall. Die von den Rezensenten gemachten Anmerkungen können direkt online im Fall angeschaut und von der den Fall einstellenden Person korrigiert werden. Erst nach Freigabe durch beide Reviewer wird der Fall veröffentlicht.

Zur Zeit der Erstellung dieses Manuskripts befindet sich die Datenbank in $\operatorname{der} \beta$-Version und ist allen Mitgliedern der AGFB zugänglich. Die Datenbank ist über den Mitgliederbereich der Homepage der DGRM erreichbar, sodass sie auch für DGRM-Mitglieder außerhalb der AGFB genutzt werden kann. Pass- wörter können dort direkt von allen Mitgliedern über einen Anfrage-Link angefordert werden.

\section{Arbeiten mit der WiFas}

\section{Einloggen}

In der $\beta$-Version der WiFas können sich Mitglieder der DGRM über den Mitgliederbereich der Internetseite der DGRM mittels eines Registrierungsformulars anmelden und erhalten so einen personifizierten Zugang zur Datenbank.

Nach dem Einloggen muss den aktuellen Datenschutzbestimmungen zugestimmt werden, bevor die Übersicht der vorhandenen Fälle erscheint. Die Datenschutzbestimmungen beinhalten u.a., dass ausschließlich die DGRM über das vollumfängliche Nutzungsrecht verfügt, die im WiFas-System zur Verfügung gestellten Inhalte (Bilder, Videos, Dokumente und Informationen) außerhalb des Systems zu verwenden bzw. für andere Personen freizugeben. Bei Nichtverwen- 


\section{Schnellsuche}

Vervenden Sie die Schnellsuche um Fälle basierend auf bestimmten Schlagvorten zu finden. Bitte trennen Sie einzelne Schlagworte mit einem Leerzeichen.

Stichwortsuche: Schlagworte eingeben suchen

\section{Graphische Suche}

Vervenden Sie die graphische Suchmaske um Fälle basierend auf bestimmten Körperregionen zu finden. Die Suche ist implizit, das heißt eine Suche auf Kopf liefert ebenfalls Ergebnisse für Nase. .

Steuerung:

Beim Überfahren des Körpers mit der Maus werden interaktive Bereiche hervorgehoben. Diese sind unterteilt in orange Detailebenen und rote Endbereiche. Detailebenen können feiner aufgelöst bzw. veiter abgestiegen verden.

Navigieren Sie zu einer geviünschten Region und markieren Sie sie mit Strg + Linksklick. Alternativ können Sie die Region auch direkt aus der nebenstehenden Auswahl selektieren.

- Linksklick: Detailebene tiefer

- Strg + Linksklick: Bereich ausvählen

- Rechtsklick: Detailebene höher

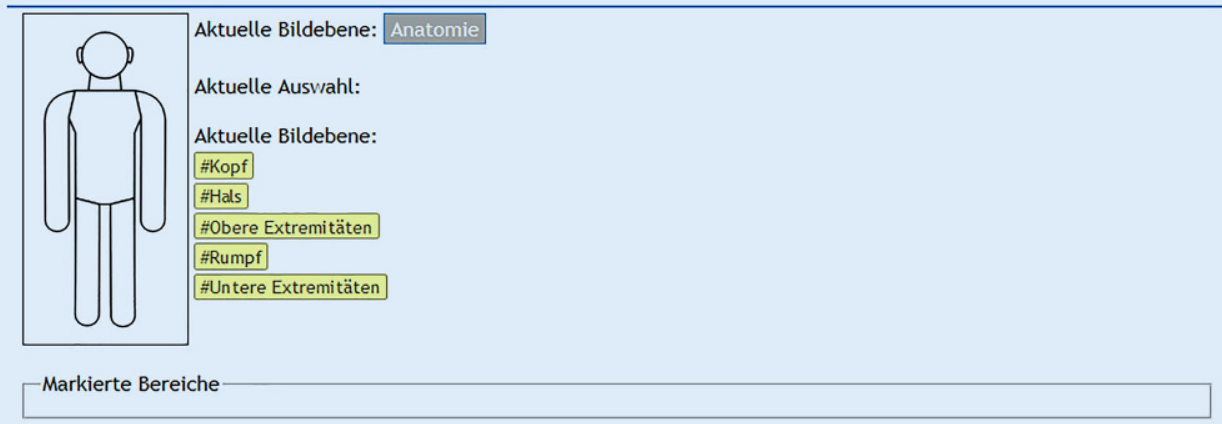

a

\section{Detailierte Suche}

Vervenden Sie die detailierte Suchmaske um Fälle basierend auf exakten Daten zu finden. Alle Eingabefelder vierden UNDVerknüpft und liefern demnach nur spezifische Fälle. Bitte trennen Sie einzelne Schlagvorte mit einem Leerzeichen.

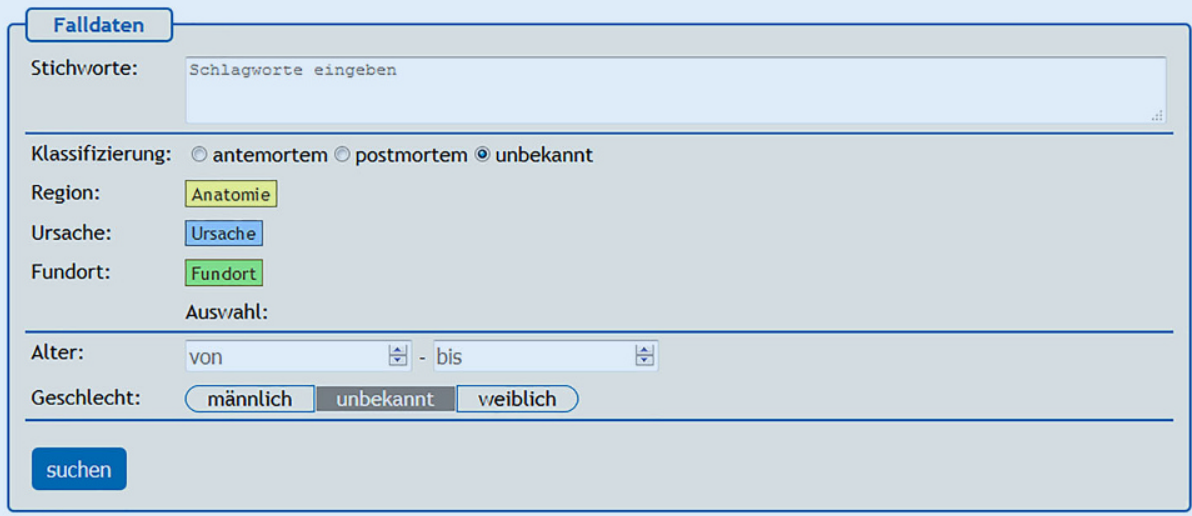

b

den der Datenbank erfolgt ein automatisches Log-out nach $23 \mathrm{~min}$.

Zur Zeit der Erstellung dieses Manuskripts können Mitglieder der DGRM über den Mitgliederbereich der Internetseite der DGRM, über eine Zwei-
Wege-Authentifizierung, einen personifizierten Zugang zum WiFas-System erhalten. Nach durchgeführter Registrierung eines neuen Nutzers erfolgt eine Legitimierung durch eine systemverantwortliche Person, welche die Registrie-
Abb. $3<$ a Suchmöglichkeit mittels Schlagwörtern und Körperregionen in der WiFas. b Suchmöglichkeit mittels Stichwörtern, Verletzungsursachen, Fundort, Alter und Geschlecht in der WiFas rung des neuen Nutzers nochmals an den Administrator betätigt.

\section{Übersicht}

In der Übersicht ist es möglich, durch die einzelnen Fälle zu scrollen und ne- 


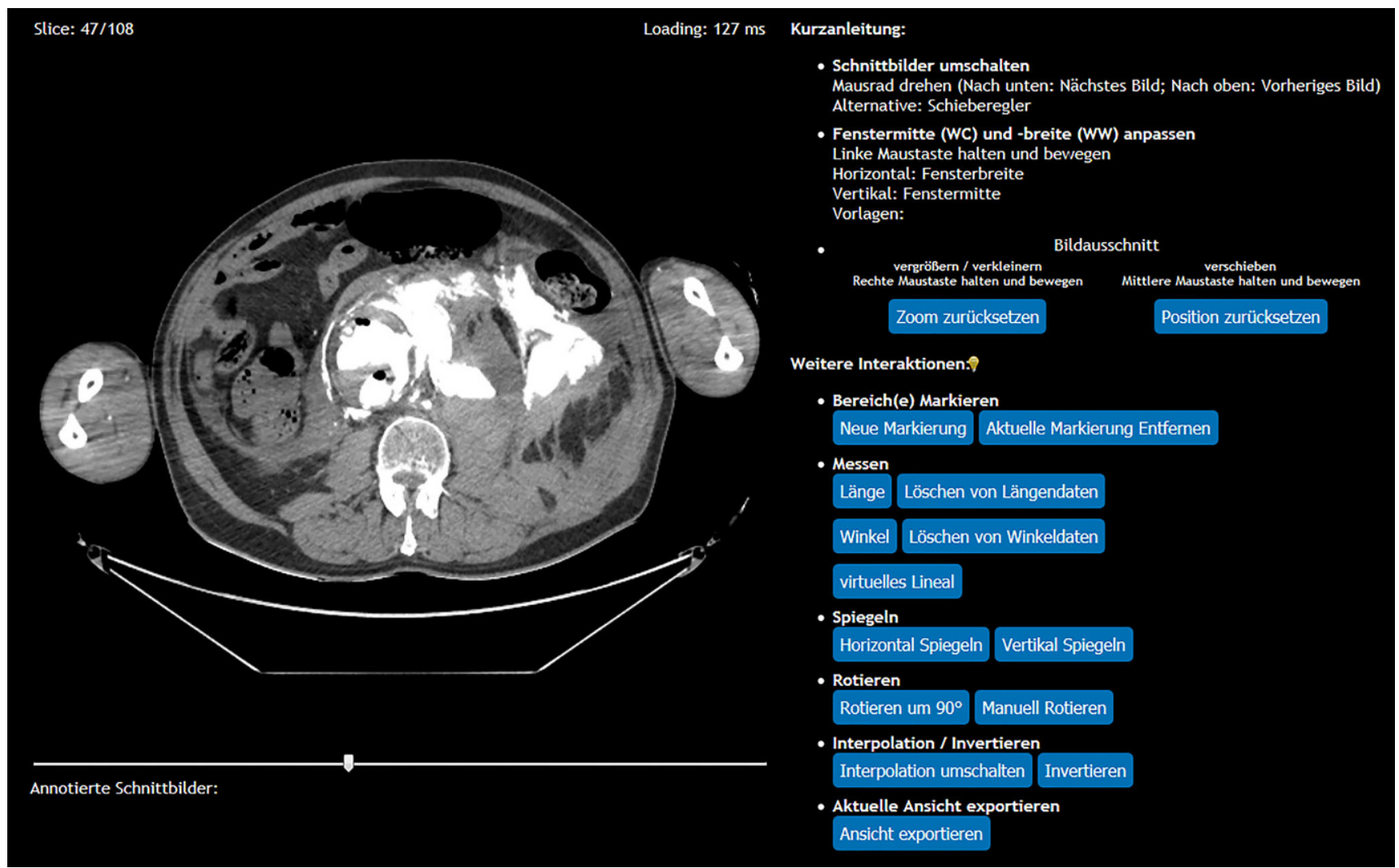

Abb. 4 A DICOM-Viewer der WiFas mit verschiedenen Funktionen (hier: Darstellung eines rupturierten Bauchaortenaneurysmas)

ben Identifikationsnummern, die bereits anzeigen, ob es sich um klinische oder postmortale Fälle handelt, erhält der Benutzer kurze Informationen zu den Fällen.

\section{Suche}

In der Taskleiste kann ferner die Suche direkt angewählt werden. Diese wiederum kann mittels Schlagwörtern, anhand von anatomischen Regionen, Fundort, Alter und Geschlecht oder angewandter Gewaltformen erfolgen (• Abb. 3a, b).

Nach Auswahl einer oder mehr Suchbegriffe werden dem Benutzer sämtliche in der Datenbank vorhandenen Fälle angezeigt, welche die zuvor festgelegten Suchkriterien erfüllen. Nun kann ein einzelner Fall ausgewählt oder die angezeigten Fälle verglichen werden.

\section{Fallauswahl}

Nach Auswahl eines Falls öffnet sich dieser mit einer allgemeinen Beschreibung, gefolgt von zu dem Fall hinterlegten Berichten und Bildmaterial.
Es können verschiedene Berichte z. B. Fundortbericht, äußere Leichenschau, Sektionsprotokoll bzw. Auszüge aus diesen oder auch klinische Untersuchungen mit entsprechender Fotodokumentation hinterlegt werden. Die Fotos enthalten erläuternde Bildunterschriften und Annotationen wichtiger Befunde, die mithilfe der Maus farblich hervorgehoben werden können.

\section{DICOM-Viewer}

Im Folgenden können dann einzelne Bilder als auch ganze DICOM-Dateien mit Schnittbildern angeschaut werden, welche die $\mathrm{zu}$ zeigenden Befunde wiedergeben. Hierbei kann eigenständig z. B. durch eine CT-Untersuchung einer Körperregion gescrollt werden. Alternativ können die Annotationen mittels Maustaste bemüht werden, um wichtige Befunde $\mathrm{zu}$ finden und ihr Erkennen $\mathrm{zu}$ erlernen (-Abb. 4). Ein radiologischer Befundbericht bei komplexeren Befunden oder bei außergewöhnlicher Beschreibung ist ebenfalls abrufbar.
Bei klinischen Fällen sind zusätzliche Untersuchungen und ggf. das weitere Vorgehen der Rechtsmedizin für den spezifischen Fall hinterlegt.

\section{Ausblick}

Geplant ist die interne Fortbildungspunktevergabe im Rahmen der Weiterbildungsakademie der DGRM für die Einstellung von neuen Fällen bzw. die Bearbeitung/Lösung von speziellen Lehrfällen für Assistenzärztinnen und -ärzte.

\section{Diskussion}

Dass die forensische Bildgebung während der letzten Jahre einen bedeutenden Stellenwert in der Rechtsmedizin eingenommen hat, zeigen u. a. zahlreiche wissenschaftliche Artikel, Kongressbeiträge als auch Lehrbücher. Sowohl die klinische forensische Bildgebung als auch die postmortale Bildgebung sind nicht mit der 
klinischen Radiologie gleichzusetzen [6, 10, 14].

Es sind die forensischen Fragestellungen sowohl bei der Erstellung des Bildmaterials als auch bei der Interpretation $\mathrm{zu}$ berücksichtigen. Dementsprechend müssen klinische CT- und MRT-Protokolle sowohl in der klinisch forensischen als auch postmortalen Bildgebung an die forensischen Bedürfnisse angepasst oder komplett neu implementiert werden. Postmortal ist mit den Leichenveränderungen eine weitere Anpassung des Protokolls für die Erstellung des Bildmaterials als auch ein Erlernen postmortaler Normalbefunde und „pitfalls“ im Vergleich zur klinischen Radiologie für die Interpretation notwendig [6]. Oftmals werden seitens der Ermittlungsbehörden zusätzlich rekonstruktive Fragen gestellt, deren Beantwortung rechtsmedizinisches Fachwissen zusätzlich zum radiologischen Wissen als auch gesonderte Rekonstruktionen der Bilddaten (z.B. VRT-Rekonstruktionen von CTDaten) erfordert. Im gesamten Gebiet der forensischen Bildgebung ist dementsprechend interdisziplinäres radiologisches und rechtsmedizinisches Wissen notwendig.

In verschiedenen bereits erschienen Artikeln und Lehrbüchern ist lediglich das Betrachten einzelner Fotografien von Befunden möglich. Das selbstständige Erkennen und eine direkte Lernkontrolle, wie sie durch die WiFas gegeben sind, können diese Bücher jedoch nicht bieten. Sie vermitteln dementsprechend nicht denselben Lernerfolg.

Mit der wissensbasierten Fallsammlung der forensischen Bildgebung wird erstmals eine Plattform angeboten, auf deren Grundlage verschiedenste Informationen aus den Bereichen der Rechtsmedizin und der Radiologie zusammenzufließen können.

In der Datenbank ist es möglich, eine Übersicht von Fällen durchzuscrollen oder gezielt nach einzelnen Fällen zu suchen. Dies kann mittels Stichwortsuche, Körperregionen, Geschlecht, Alter oder Gewaltarten erfolgen. Der Vergleich sämtlicher Fälle, die den Suchkriterien entsprechen, ist so möglich. In einem Fall bekommt der Leser zunächst einen Überblick, wird dann durch verschiedene hin- terlegte Dokumente wie z. B. den Fundortbericht, die äußere Leichenschau mit Fotos und Annotationen der Fotos zu der Sektion und den radiologischen Untersuchungen geführt. Zum Beispiel könnte neben der Sektion ein postmortales CT vorliegen, das im DICOM-Viewer selbst auf wichtige Befunde durchgeschaut werden kann. Zur Verbesserung des Lerneffekts ist es möglich, hier Markierungen und Beschreibungen mittels der Maus zu erhalten. Diese radiologischen Befunde können wiederum mit den Sektionsbefunden verglichen werden. Idealerweise befinden sich zusätzlich der radiologische Befundbericht und Auszüge aus dem Sektionsprotokoll als Datei im Anhang, sodass die Verschriftlichung der Befunde direkt mitgelernt werden kann.

Das Hochladen von Fällen in die WiFas bedarf, ähnlich dem Hochladen eines Artikels in einer Fachzeitschrift, einiger Vorbereitung. So müssen z.B. die verschiedenen Berichte anonymisiert werden. Ferner müssen sehr große DICOMDatensätze auf wesentliche Bereiche beschränkt werden (z. B. CT-Abdomen anstelle eines Ganzkörper-Scans). Fehler oder Probleme mit der Benutzung der WiFas können direkt in dieser an den Administrator gemeldet werden. Sollte es zu systematischen Problemen mit der Anwenderfreundlichkeit der Datenbank kommen, können diese hierdurch bereits vor der geplanten Evaluation (s. unten) zeitnah behoben werden.

So kann benutzerindividuell je nach bereits bestehendem Wissen bzw. notwendiger Intensität des Lernens Fach- wissen zu verschiedenen Fragestellungen im Bereich der forensischen Bildgebung von jedem Mitglied der DGRM erworben bzw. vertieft werden.

Unseres Wissens existiert bislang keine weitere solche Datenbank für forensische Bildgebung.

Dementsprechend hat die WiFas für alle Mitglieder der DGRM die Möglichkeit geschaffen, individuell forensische Bildgebung zu lernen und sich für die Verwendung der Bildgebung in der täglichen Routine vorzubereiten. Dies gewährt ein systematisches und fundiertes Erlernen der forensischen Bildgebung unabhängig davon, wie viele Untersuchungen im eigenen Institut durchgeführt werden.

Um diesen Lerneffekt zu evaluieren, ist die Durchführung einer Fragebogenstudie nach 1,5 Jahren geplant. Direkt online bei Zugriff auf die WiFas wird dem Nutzer einmalig ein standardisierter Fragebogen vorgelegt, in dem Punkte zum Aufbau und zur Anwenderfreundlichkeit der WiFas sowie zum eigenen Verwenden und zum Lernen abgefragt werden. Die Ergebnisse werden nach Abschluss der Befragung veröffentlicht.

\section{Fazit für die Praxis}

Die WiFas schließt auf interaktive Art und Weise die Lücke zwischen wachsenden Ansprüchen an forensisch-radiologischen Wissen und der derzeit noch limitierten Verbreitung desselben. Sie erlaubt somit Rechtsmedizinerinnen und Rechtsmedizinern der DGRM, sich
Hier steht eine Anzeige.

$$
\text { Springer }
$$


auf die Verwendung der forensischen Bildgebung vorzubereiten oder sich bei bereits etablierter Anwendung darin zu verbessern.

\section{Korrespondenzadresse}

\section{Dr. med. S. Heinze}

Institut für Rechts- und Verkehrsmedizin, Universitätsklinikum Heidelberg

Heidelberg, Deutschland

sarah.heinze@med.uni-heidelberg.de

Funding. Open Access funding provided by Projekt DEAL.

\section{Einhaltung ethischer Richtlinien}

Interessenkonflikt. S. Heinze, S. Grunert, S. Grabherr und D. Labudde geben an, dass kein Interessenkonflikt besteht.

Für diesen Beitrag wurden von den Autoren keine Studien an Menschen oder Tieren durchgeführt. Für die aufgeführten Studien gelten die jeweils dort angegebenen ethischen Richtlinien.

Open Access. Dieser Artikel wird unter der Creative Commons Namensnennung 4.0 International Lizenz veröffentlicht, welche die Nutzung, Vervielfältigung Bearbeitung, Verbreitung und Wiedergabe in jeglichem Medium und Format erlaubt, sofern Sie den/die ursprünglichen Autor(en) und die Quelle ordnungsgemäß nennen, einen Link zur Creative Commons Lizenz beifügen und angeben, ob Änderungen vorgenommen wurden.

Die in diesem Artikel enthaltenen Bilder und sonstiges Drittmaterial unterliegen ebenfalls der genannten Creative Commons Lizenz, sofern sich aus der Abbildungslegende nichts anderes ergibt. Sofern das betreffende Material nicht unter der genannten Creative Commons Lizenz steht und die betreffende Handlung nicht nach gesetzlichen Vorschriften erlaubt ist, ist für die oben aufgeführten Weiterverwendungen des Materials die Einwilligung des jeweiligen Rechteinhabers einzuholen.

Weitere Details zur Lizenz entnehmen Sie bitte der Lizenzinformation auf http://creativecommons.org/ licenses/by/4.0/deed.de.

\section{Literatur}

1. Bornik A, Heinze S, Campana L et al (2019) Theoretische Grundlagen der forensischen Bildgebung Rechtsmedizin 29:1-12

2. Chevallier C, Doenz F, Vaucher P et al (2013) Postmortem computed tomography angiography vs. conventional autopsy: advantages and inconveniences of each method. Int J Legal Med 127:981-989

3. Flach PM, Thali MJ, Germerott T (2014) Times have changed! Forensic radiology - a new challenge for radiology and forensic pathology. AJR Am J Roentgenol 202:W325-34

4. Glemser P, Krauskopf A, Simons D et al (2015) Klinisch-forensische Bildgebung - Erfassung und Dokumentation innerer Verletzungsbefunde bei lebenden Gewaltopfern. Rechtsmedizin 25:67-80
5. Grabherr S (2014) Gründung der Arbeitsgemeinschaft Forensische Bildgebung der DGRM. Rechtsmedizin 24:537

6. Grabherr S, Wittig H, Dedouit F et al (2015) Pitfalls in post-mortem CT-angiography-intravascular contrastinduces post-mortem pericardial effusion. Leg Med 17:218-219

7. Grabherr S, Egger C, Vilarino Ret al (2017) Modern post-mortem imaging: an update on recent developments. Forensic Sci Res 2:52-64

8. Heinze S, Uebbing K, Schroeder RJ et al (2014) Conventional radiology versus the increasing importance of post-mortem computed tomography. Arch Kriminol 234(3-4):127-133

9. Heinze S, Schröder RJ, Herbig J et al (2016) Analysis of metacarpal $V$ fractures of an interdisciplinary emergency room of an urban German hospital from a forensic viewpoint. Aust J Forensic Sci 48:445-453

10. Heinze S, Hettler L, Breitmeier D et al (2019) Postmortale Befundung thorakaler Röntgenbilder - Fachärzte für Rechtsmedizin und Radiologie im Vergleich. Arch Kriminol 243:28-38

11. Jackowski C, Warntjies MJ, Kihlberg J et al (2011) Quantitative MRI in isotropic spatial resolution for forensic soft tissue documentation. Why and how? J Forensic Sci 56:208-215

12. Kettner M, Ramsthaler F, Grabherr S et al (2017) Forensische Bildgebung im deutschsprachigen Raum - eine Bestandsaufnahme. Rechtsmedizin 27:407-413

13. Lerdorf R (2007) PHP's design goal from the very beginning is very simple. To solve the common web problem. That's it. In: Lerdorf R, Tatroe K, Maclntyre $P$ (Hrsg) Programmieren mit PHP. O'Reilly, Köln

14. O'Donnell C, Woodford N (2008) Post-mortem radiology - a new sub-speciality? Clin Radiol 63(11):1189-1194

15. Ramsthaler F, Kettner M, Birngruber CG (2018) Evidential power and explanatory gap in forensic imaging. Rechtsmedizin 28:33-40

16. Thali MJ, Dirnhofer R (2004) Forensic radiology in German-speaking area. Forensic Sci Int 144:233-242

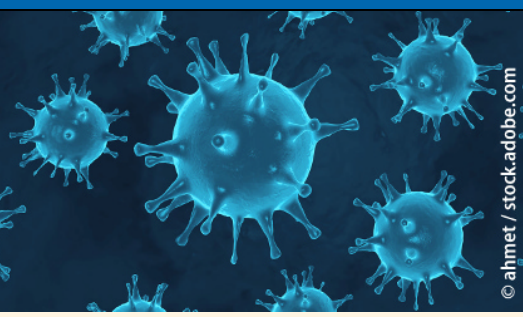

\section{Aktuelle Entwicklung zu COVID-19 bei Springer Nature und Springer Medizin}

Springer Nature und Springer Medizin unterstützen die globale Reaktion auf die COVID-19-Pandemie, in dem ein schneller und direkter $\mathrm{Zu}$ gang zu den neuesten verfügbaren Forschungsergebnissen und Daten ermöglicht wird.

Auf der Homepage SpringerMedizin.de finden Sie ein immer aktuelles Dossier mit Beiträgen, Forschungsarbeiten und Ergebnissen zu SARS-CoV-2 sowie relevanten Links.

Darin z.B. auch die kürzlich publizierte

Empfehlung von DIVI, DGIIN, DGAI und DGP zur Intensivtherapie von Patienten mit COVID-19.

Springer Nature arbeitet mit globalen Organisationen zusammen, und verlinkt über SpringerNature.com/de auf eine eigene Landingpage mit einer Vielzahl an Information sowie freiem Zugriff auf die COVID-19-Contentplattformen von Nature Research, BioMed Central (BMC) und Springer.

Das Dossier zu Coronavirus / Covid-19 von Springer Medizin finden Sie hier

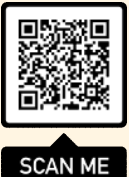

